\title{
Level alignment of gap state at organic-metal interface
}

\author{
M. Aoki, ${ }^{1, a)}$ S. Toyoshima, ${ }^{2}$ T. Kamada, ${ }^{1}$ M. Sogo, ${ }^{1}$ S. Masuda, ${ }^{1}$ T. Sakurai, ${ }^{2}$ and \\ K. Akimoto ${ }^{2}$ \\ ${ }^{1}$ Department of Basic Science, Graduate School of Arts and Sciences, The University of Tokyo, Komaba, \\ Meguro, Tokyo 153-8902, Japan \\ ${ }^{2}$ Institute of Applied Physics, University of Tsukuba, 1-1-1 Tennodai, Tsukuba, Ibaraki 305-8573, Japan
}

(Received 21 May 2009; accepted 16 July 2009; published online 28 August 2009)

\begin{abstract}
Electron emission spectra resulting from thermal collision of $\operatorname{He}^{*}\left(2^{3} S\right)$ atoms with 2,9-demethyl-4,7-diphenyl-1,10-phenanthroline (BCP) films deposited on metal substrates were measured to characterize gap states emerged at the organic-metal interface. For BCP on Au, the gap state is originated from weak chemisorption and serves as a mediator of metal wave functions to the first layer. For BCP on K, organic-metal complex is formed by spontaneous diffusion, yielding the gap states delocalized over the film. In the interfacial region, all the gap state reveals an incommensurate shift with the valence band top of the film, indicating the breakdown of the Schottky-Mott model as evaluating the transport characteristics in organic-metal system. (C) 2009 American Institute of Physics. [DOI: 10.1063/1.3204663]
\end{abstract}

\section{INTRODUCTION}

The electronic properties of functional organic films on a metal substrate are of great interest for the basic understanding and for the potential applications to molecule-based devices such as organic light emitting diode (OLED), organic film solar cell (OFSC), etc. ${ }^{1-4}$ One of the critical issues in the field is to control the gap states, ${ }^{5,6}$ especially those emerged near the Fermi level $\left(E_{F}\right)$ of the substrate. When the organic molecules are chemically bound to a metal substrate accompanied by charge transfer, the resulting gap state would mediate the extension of metal wave functions to the organic film. Such a chemisorption-induced gap state is also responsible for the formation of dipole layer at the organic-metal interface, leading to the change in the charge injection barrier. $^{7,8}$ If an organic-metal complex (organometallic compound) is formed by coevaporation (or spontaneous diffusion of substrate atoms into the deposited film), the resulting gap state would be distributed over the film to determine the charge injection efficiency, conductivity of the film, band bending in the space charge region, ${ }^{9,10}$ etc. This type of gap state is denoted here as complex-based gap state. Thus the systematic understanding and controlling of the gap state is a key factor for fabricating novel organic-metal systems, as in the case of heterojunctions in semiconductor devices, ${ }^{11}$ but the experimental information remains rather limited.

Metastable atom electron spectroscopy (MAES) used here is based on energy analysis of emitted electrons by thermal collision of rare gas metastable atoms such as $\mathrm{He}^{*}\left(1 s 2 s, 2^{3} S\right)$ with a solid surface. ${ }^{12,13}$ On an insulating organic film, the metastable atoms decay via Penning ionization, where a valence electron of the film fills the $\mathrm{He}^{*} 1 s$ hole and the $2 s$ electron is emitted simultaneously. This process yields a single-hole spectrum as in the case of ultraviolet photoemission spectroscopy (UPS). Since the metastable atoms do not penetrate into the bulk, the local electronic states

${ }^{\text {a)} E l e c t r o n i c ~ m a i l: ~ c m a o k i @ m a i l . e c c . u-t o k y o . a c . j p . ~}$ at the outermost layer are selectively probed. This enables us to detect directly the chemisorption-induced states near $E_{F}$, e.g., $2 \pi^{*}$ state for $\mathrm{CO}$ on $\mathrm{Ni}(111)^{14}$ and antibonding $\pi$ state for $\mathrm{C}_{6} \mathrm{H}_{6}$ on $\mathrm{Pd}(110),{ }^{15}$ and to trace the hole response at the topmost layer of condensed film. ${ }^{16,17}$

In this paper we have taken up 2,9-demethyl-4,7diphenyl-1,10-phenanthroline (BCP) (Fig. 1) films on polycrystalline $\mathrm{Au}$ (a typical electrode in organic devices) and $\mathrm{K}$-precovered $\mathrm{Au}$ substrate, as a model system. The BCP films have been widely used as an electron transport material in OLED and OFSC. ${ }^{18-22}$ According to the UPS studies for BCP on metal substrates, the Schottky-Mott model ${ }^{11}$ breaks down in the sense that the vacuum level does not align across the organic-metal interface due to the presence of dipole layer. The gap states have been observed in BCP films on low work function metals ${ }^{23,24}$ and Li-doped BCP film. ${ }^{25}$ The purpose of the present study is twofold. One is to identify two types of gap states by probing the local electronic states emerged at the topmost layer. Another purpose is to clarify the level alignment of the gap states as a function of film thickness. In the interfacial region, all the gap state shows an incommensurate shift with the valence band top of the films. This finding indicates that the Schottky-Mott model is not valid as evaluating the charge transport in organic-metal system.

\section{EXPERIMENT}

All experiments were performed by an ultrahigh vacuum spectrometer. $^{26,27}$ The polycrystalline $\mathrm{Au}$ substrate was cleaned by repeated $\mathrm{Ar}^{+}$ion sputtering and heating cycles. The clean substrate showed no impurities within the detection limit of Auger electron spectroscopy. Potassium was deposited from a SAES getter source onto the clean substrate and the thickness was estimated to several monolayers. The BCP films were prepared by vacuum deposition and the film thickness was monitored with a quartz oscillator calibrated in advance. 

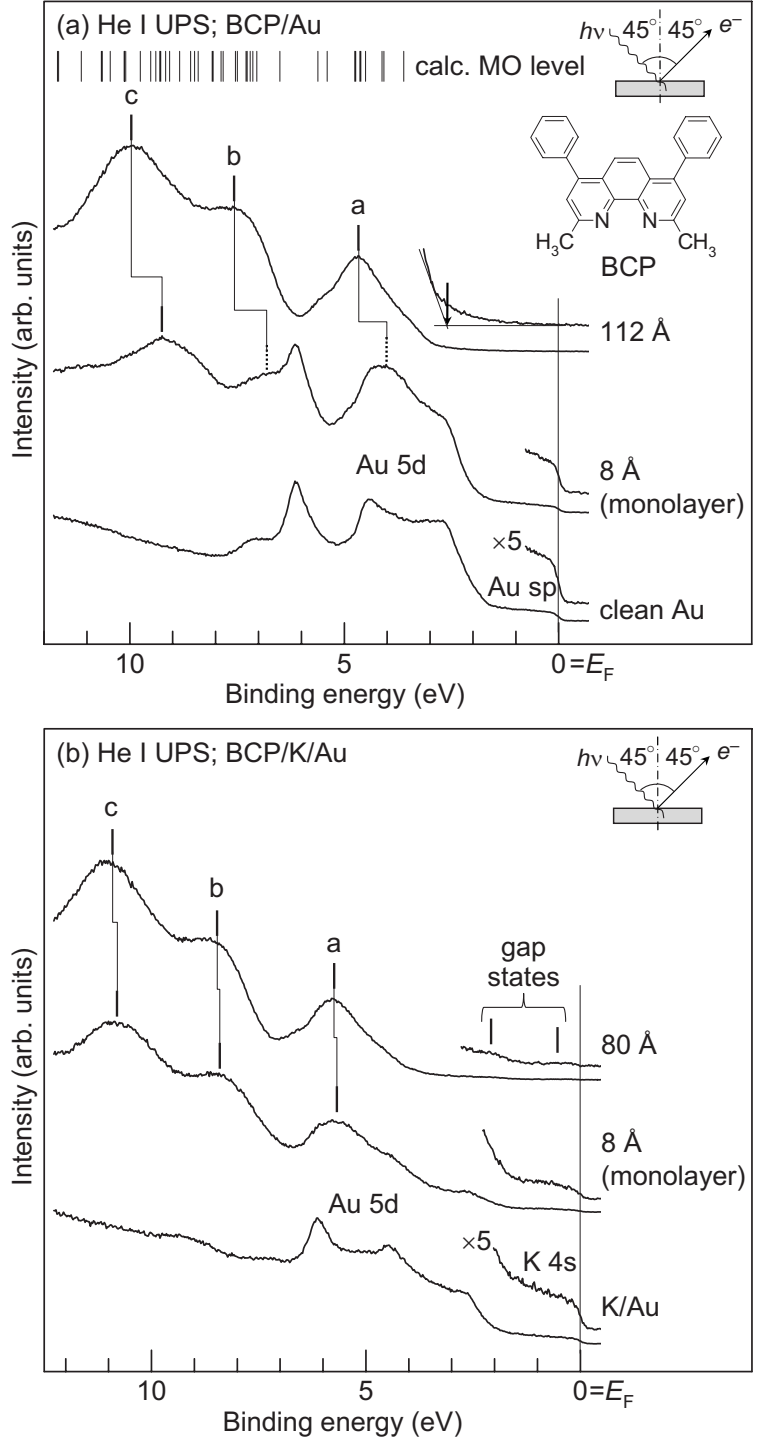

(c) BCP/Au

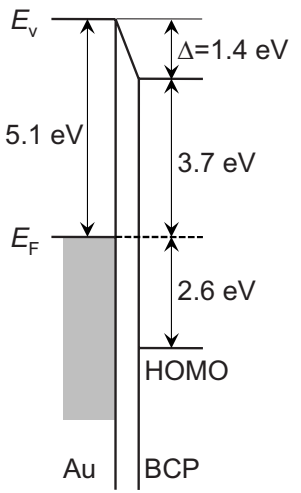

(d) $\mathrm{BCP} / \mathrm{K} / \mathrm{Au}$
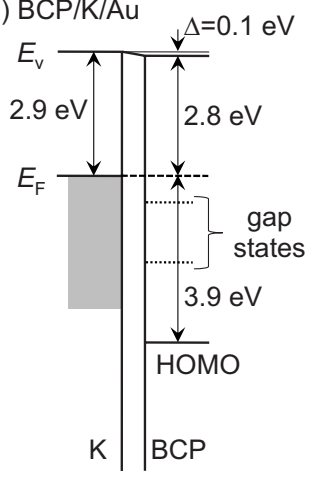

FIG. 1. He I UPS spectra of BCP films deposited on (a) polycrystalline Au and (b) K-precovered Au substrate. (c) and (d) show the corresponding energy level diagrams for bulk BCP.

\section{RESULTS AND DISCUSSIONS}

Fig. 1(a) shows the He I UPS spectra of BCP films deposited on polycrystalline $\mathrm{Au}$ at room temperature. The binding energy $\left(E_{B}\right)$ is referred to the Fermi level $\left(E_{F}\right)$ of the substrate. The nominal thickness of 6-8 $\AA$ corresponds to monolayer on the average. The substrate bands ( $s p$ band just below $E_{F}$ and higher-lying $5 d$ bands) attenuate upon deposition, accompanied by the appearance of broad BCP-derived bands labeled a-c. The valence levels of BCP obtained by molecular orbital (MO) calculation using density functional theory are shown in Fig. 1(a). It shows that the lowest-lying band $\mathrm{a}$ is assigned to ten MOs, in which the highest occupied MO (HOMO) is composed of phenathroline $\pi$ orbital. With increasing film thickness, the BCP-derived bands reveal a characteristic shift to the higher $E_{B}$. The overall features, including the vacuum level shift $(\Delta)$ determined by secondary electron cutoff (not shown), are essentially identical with our early data. ${ }^{23}$ The level parameters for bulk BCP (112 $⿱$ ) on $\mathrm{Au}$ are shown in Fig. 1(c). The ionization energy of bulk $\mathrm{BCP}$, defined as the energy difference between the leading edge of band a (indicated by an arrow in the spectrum) and the vacuum level, is $6.3 \mathrm{eV}$, which is in agreement with the values in literature. ${ }^{18,28}$ In Fig. 1(b), the He I UPS spectra of BCP films deposited on K-precovered Au substrate are shown, together with the level diagram for $80 \AA$ BCP. The diagram (Fig. 1(d)) shows that the vacuum level slightly shifts downward and the HOMO state is located at the high $E_{B}$ compared to the case of BCP on Au. The dotted levels at 0.6 and $2.0 \mathrm{eV}$ below $E_{F}$ are attributed to gap states induced in the HOMO-lowest unoccupied MO (LUMO) gap of BCP (see below). In both systems, however, it is not easy to trace the BCP-derived states near $E_{F}$ due to heavy overlap with the substrate bands, especially during the formation of electric double layer.

To clarify the local electronic states near $E_{F}$, we measured the $\mathrm{He}^{*}\left(2^{3} S\right)$ MAES spectra of BCP films on the clean and K-precovered Au substrate prepared under the same conditions. The typical data are shown in Figs. 2(a) and 2(b), respectively. The $\mathrm{He}^{*}\left(2^{3} S\right)$ atoms de-excite on the Au substrate dominantly via resonance ionization (RI) followed by Auger neutralization (AN), yielding two-hole states located above $E_{B} \approx 2 \mathrm{eV}$ in the spectrum. A weak band just below $E_{F}$ is attributed to Penning ionization (PI) occurring as a competing process and its detail will be described elsewhere. ${ }^{29}$ Upon deposition of $\mathrm{BCP}$, the $\mathrm{RI}+\mathrm{AN}$ process is gradually suppressed by the coverage of the Au surface, whereas the PI process becomes dominant to yield one-hole state as in photoemission. The PI process also takes place in the BCP-K system, reflecting the low work function of the $\mathrm{K}$ layers. ${ }^{12}$ It turns out from Figs. 2(a) and 2(b) that the BCPderived bands are clearly observed even in the submonolayer region, since the PI process takes place selectively at the topmost layer. A tail part of band a in the lower $E_{B}$ is attributed to the HOMO-derived state by comparison with the calculated MO levels. Furthermore, weak emissions near $E_{F}$ are attributable to the gap states.

At first we characterize two types of gap states, based on the MAES spectra. For BCP on Au, a weak band appears just below $E_{F}$, whose intensity grows during the monolayer $(8 \AA)$ formation and then decreases during the second layer $(16 \AA)$ formation. Therefore, the gap state is highly localized at the Au-BCP boundary and classified into the chemisorptioninduced type. The gap state is originated probably from weak coupling between BCP LUMO and Au sp band, taking the HOMO level in bulk BCP $\left(E_{B}=3.4 \mathrm{eV}\right)$ and the optical 

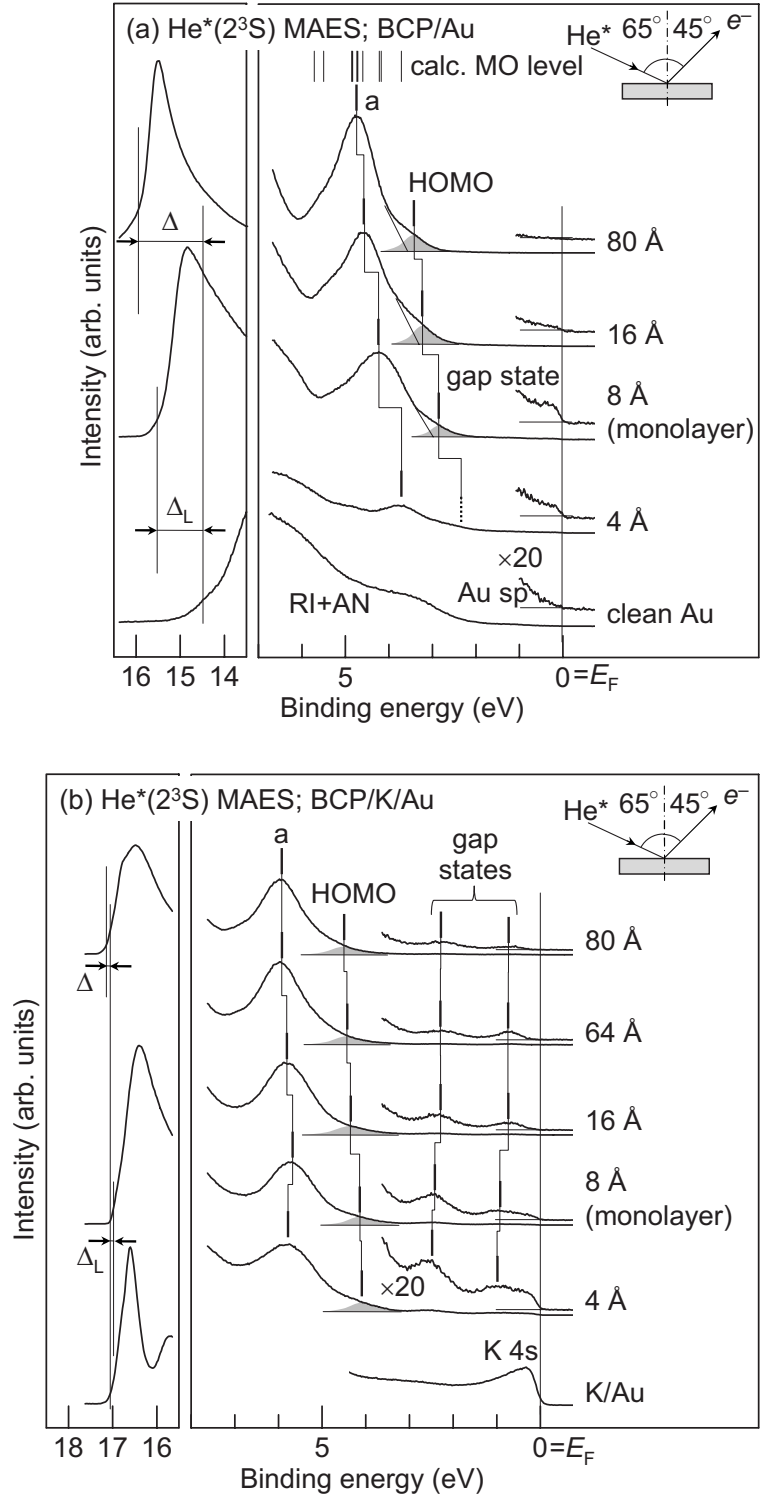

FIG. 2. $\mathrm{He}^{*}\left(2^{3} S\right)$ MAES spectra of BCP films deposited on (a) polycrystalline $\mathrm{Au}$ and (b) K-precovered Au substrate. The vacuum level shift is indicated by $\Delta$.

HOMO-LUMO gap $(3.5 \mathrm{eV})^{23}$ into account. It is known that some electron-acceptor molecules such as tetrafuluorotetracyanoquinodimethane are chemically bound to gold surface to yield the gap state near $E_{F}{ }^{6}$ Furthermore, the gap state reveals a clear Fermi edge in the spectra, indicating that the electronic properties of $\mathrm{BCP}$ on $\mathrm{Au}$ are suddenly altered from the metallic to insulating phases, on going from the first layer to the subsequent layers. A similar phase change has been observed in the MAES spectra of alkanethiol on $\operatorname{Pt}(111),{ }^{27,30}$ benzenethiol on $\operatorname{Pt}(111),{ }^{31}$ etc.

Another type of gap state can be identified in $\mathrm{BCP}$ on $\mathrm{K}$-precovered Au. As is seen in Fig. 2(b), two gap states emerge near $E_{F}$ irrespective of layer thickness, in striking contrast to the case of BCP on Au. For $64 \AA$ BCP, the gap states are located at 0.6 and $2.2 \mathrm{eV}$ below $E_{F}$, which correspond well to those observed in the UPS spectrum. Therefore, it is evident that the gap states are widely distributed over the film, due to spontaneous diffusion of $\mathrm{K}$ atoms into (a) $\mathrm{BCP} / \mathrm{Au}$

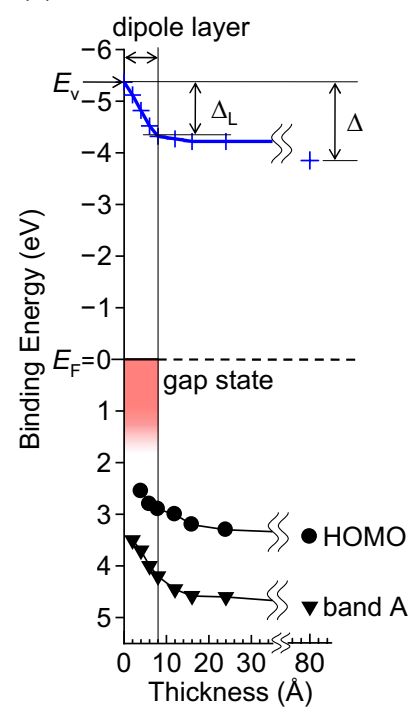

(b) $\mathrm{BCP} / \mathrm{K} / \mathrm{Au}$

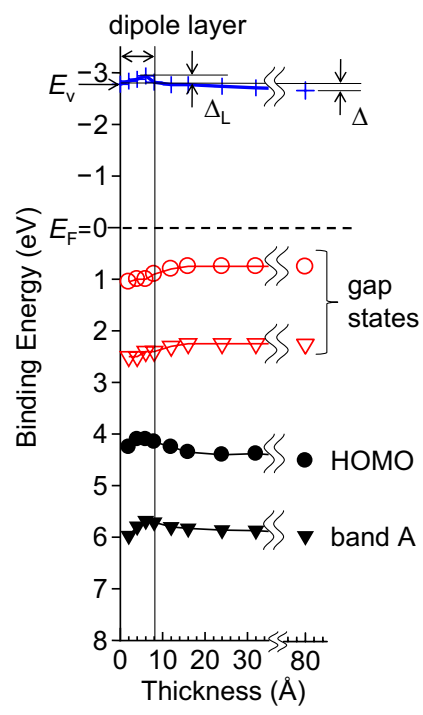

FIG. 3. (Color online) Energy level diagrams (referred to $E_{F}$ ) of BCP valence bands including gap states as a function of film thickness. Vacuum level shifts determined by the cutoff energy of true secondary in the MAES spectra are also shown in the figure.

the BCP layers and subsequent formation of organic-metal complex (organometallic compound). In this sense, the gap states would be classified into the complex-based type. A similar gap state was observed in the UPS spectra of Lideposited BCP films. ${ }^{25}$ Our data also show that the K $4 s$-derived band just below $E_{F}$ is missing in thick films. This suggests that the gap state at $0.6 \mathrm{eV}$ is derived from electron transfer from $\mathrm{K} 4 s$ to LUMO of $\mathrm{BCP}$, whereas the higherlying gap state is due to split-off state of BCP HOMO (although the theoretical calculations are necessary for the detail assignment). Due to the lack of the Fermi edge, the gap states are semiconducting in nature, which is also in contrast to the case of BCP on Au.

Next, we examine the changes in the binding energy depending on the film thickness, which is shown in Fig. 3. The vacuum level shifts determined by the cutoff energy of true secondary in the MAES spectra [Figs. 2(a) and 2(b)] are also shown in the figure, which are essentially identical with those by the UPS spectra. For BCP on Au, the vacuum level shifts downward steeply until coverage of the first layer, and then slightly during the layer growth. This indicates that the vacuum level shift is caused predominantly by the local electric properties at the BCP-Au boundary, as in ordinary organic-metal systems. ${ }^{7}$ To clarify the contribution of the organic-metal boundary, the local shift $\left(\Delta_{L}\right)$, defined as the vacuum level shift during the monolayer formation, is newly introduced in Fig. 3. The observed $\Delta_{L}$ and $\Delta$ are 1.0 and 1.5 $\mathrm{eV}$, respectively. As is seen in Fig. 3(a), the BCP-derived bands (HOMO and top of band a) shift downward in a similar manner, whereas the gap state is pinned at $E_{F}$ due to its metallic nature. During the second layer formation, the $\mathrm{He}^{*}\left(2^{3} S\right)$ atoms collide with the topmost BCP molecules in the second layer and in the uncovered first layer. Therefore, the observed binding energy is averaged, giving rise to an apparent shift without a clear inflection point. As a conse- 
quence, no indication of band bending is found in the BCP film on $\mathrm{Au}$, reflecting the insulating nature of $\mathrm{BCP}$ bulk mentioned above.

As for BCP-K system, the vacuum level slightly shift upward until monolayer and then slightly downward with increasing layer thickness. The observed $\Delta_{L}$ and $\Delta$ are -0.2 and $0.1 \mathrm{eV}$, respectively. The upward local shift in $\Delta_{L}$ indicates the formation of a reverse dipole layer as in the case of $\mathrm{BCP}$ on Au. The change in the binding energy of the BCPderived states (HOMO and top of band a) corresponds well to the vacuum level shift. As is seen in Fig. 3(b), the gap states reveal apparently different shift during multilayer formation. This is caused by the fact that the gap states are delocalized over the film and the binding energies depend on the layer thickness, in contrast to the case of the HOMO localized in BCP.

Finally we address briefly the relationship between the energy diagram and charge injection efficiency at the organic-metal interface. The hole injection from a metal electrode to an organic film strongly depends on the injection barrier formed at the interface, which is defined by energy difference between the Fermi level of the electrode and the valence band top of the film. In the Au-BCP system, the barrier height at the Au-BCP boundary is zero owing to the presence of the metallic gap state. However, it attains to $2 \mathrm{eV}$ or more at the interface between the first and second layer, reflecting the metal-insulator phase change. As a consequence, the chemisorption-induced gap state plays a minor role in the overall injection efficiency in the metal-organic system. On the other hand, the injection barrier in the K-BCP system becomes low owing to the presence of the delocalized gap states, although it depends on the film thickness. In addition, the semiconducting nature of the K-BCP system would improve the charge transport characteristics.

\section{SUMMARY}

We demonstrate that MAES is useful tool to characterize the gap state emerged at organic-metal interface. For BCP on $\mathrm{Au}$, the chemisorption-induced gap state appears at the organic-metal boundary and serves as a mediator of the extension of metal wave functions to the film. Also the complex-based gap states were identified in the BCP-K system. In the interfacial region, all the gap state shows an incommensurate shift with the valence band top of the film, indicating the breakdown of the Schottky-Mott model as evaluating the charge transport in organic-metal system.

\section{ACKNOWLEDGMENTS}

This study is financially supported by Grant-in-Aid for Scientific Research on Priority Areas (No. 17069007) from MEXT.

${ }^{1}$ M. A. Baldo, D. F. O’Brien, Y. You, A. Shoustikov, S. Sibley, M. E. Thompson, and S. R. Forrest, Nature (London) 395, 151 (1998).

${ }^{2}$ G. Schwartz, K. Fehse, M. Pfeiffer, K. Walzer, and K. Leo, Appl. Phys. Lett. 89, 083509 (2006).

${ }^{3}$ M. Granström, K. Petritsch, A. C. Arias, A. Lux, M. R. Andersson, and R. H. Friend, Nature (London) 395, 257 (1998).

${ }^{4}$ P. Peumans, S. Uchida, and S. R. Forrest, Nature (London) 425, 158 (2003).

${ }^{5}$ S. Masuda, H. Hayashi, Y. Harada, and S. Kato, Chem. Phys. Lett. 180, 279 (1991).

${ }^{6}$ N. Koch, S. Duhm, J. P. Rabe, A. Vollmer, and R. L. Johnson, Phys. Rev. Lett. 95, 237601 (2005)

${ }^{7}$ H. Ishii, K. Sugiyama, E. Ito, and K. Seki, Adv. Mater. 11, 605 (1999).

${ }^{8}$ N. Koch, J. Phys.: Condens. Matter 20, 184008 (2008).

${ }^{9}$ S. Braun, W. Osikowicz, Y. Wang, and W. R. Salaneck, Org. Electron. 8, 14 (2007).

${ }^{10}$ W. Gao and A. Kahn, J. Appl. Phys. 94, 359 (2003).

${ }^{11}$ S. M. Sze and K. K. Ng, Physics of Semiconductor Devices (Wiley, New York, 2007).

${ }^{12}$ Y. Harada, S. Masuda, and H. Ozaki, Chem. Rev. (Washington, D.C.) 97, 1897 (1997).

${ }^{13}$ H. Morgner, Adv. At., Mol., Opt. Phys. 42, 387 (2000).

${ }^{14}$ F. Bozso, J. T. Yates, Jr., J. Arias, H. Metiu, and R. M. Martin, J. Chem. Phys. 78, 4256 (1983).

${ }^{15}$ J. Yoshinobu, M. Kawai, I. Imamura, F. Marumo, R. Suzuki, H. Ozaki, M. Aoki, S. Masuda, and M. Aida, Phys. Rev. Lett. 79, 3942 (1997).

${ }^{16}$ Y. Harada, H. Ozaki, and K. Ohno, Phys. Rev. Lett. 52, 2269 (1984).

${ }^{17}$ R. Suzuki, H. Taoka, M. Aoki, S. Masuda, and Y. Morikawa, Phys. Rev. B 65, 035416 (2001).

${ }^{18}$ Y. Kijima, N. Asai, and S. Tamura, Jpn. J. Appl. Phys., Part 1 38, 5274 (1999).

${ }^{19}$ J. Tardy, M. Ben Khalifa, and D. Vaufrey, Mater. Sci. Eng., C 26, 196 (2006).

${ }^{20}$ Q. L. Song, F. Y. Li, H. Yang, H. R. Wu, X. Z. Wang, W. Zhou, J. M. Zhao, X. M. Ding, C. H. Huang, and X. Y. Hou, Chem. Phys. Lett. 416, 42 (2005).

${ }^{21}$ J. Xue, S. Uchida, B. P. Rand, and S. R. Forrest, Appl. Phys. Lett. 85, 5757 (2004).

${ }^{22}$ T. Taima, S. Toyoshima, K. Hara, K. Saito, and K. Yase, Jpn. J. Appl. Phys., Part 2 45, L217 (2006).

${ }^{23}$ S. Toyoshima, K. Kuwabara, T. Sakurai, T. Taima, K. Saito, H. Kato, and K. Akimoto, Jpn. J. Appl. Phys., Part 1 46, 2692 (2007).

${ }^{24}$ S. Toyoshima, T. Sakurai, T. Taima, K. Saito, H. Kato, and K. Akimoto, Jpn. J. Appl. Phys. 47, 1397 (2008).

${ }^{25}$ G. Parthasarathy, C. Shen, A. Kahn, and S. R. Forrest, J. Appl. Phys. 89, 4986 (2001)

${ }^{26}$ M. Aoki, Y. Ohashi, S. Masuda, S. Ojima, and N. Ueno, J. Chem. Phys. 122, 194508 (2005).

${ }^{27}$ M. Aoki, Y. Koide, and S. Masuda, J. Electron Spectrosc. Relat. Phenom. 156-158, 383 (2007).

${ }^{28}$ I. G. Hill and A. Kahn, J. Appl. Phys. 86, 4515 (1999).

${ }^{29}$ S. Masuda, K. Sasaki, M. Sogo, M. Aoki, and Y. Morikawa (to be published).

${ }^{30}$ S. Masuda, Y. Koide, M. Aoki, and Y. Morikawa, J. Phys. Chem. C 111, 11747 (2007)

${ }^{31}$ S. Masuda, K. Sasaki, M. Aoki, and Y. Morikawa (to be published). 